\title{
Acurácia da Reanálise ERA-Interim do ECMWF e sua Aplicação na Estimativa da Deficiência Hídrica no Estado do Paraná, Brasil
}

\author{
Lucas Eduardo de Oliveira Aparecido ${ }^{1}$ (D), Glauco de Souza Rolim ${ }^{2}$, \\ Jose Reinaldo da Silva Cabral de Moraes ${ }^{1}$, Guilherme Botega Torsoni ${ }^{1}$, \\ Kamila Cunha de Meneses ${ }^{2}$, Cicero Teixeira Silva Costa $^{1}$ \\ ${ }^{1}$ Instituto Federal de Educação, Ciência e Tecnologia de Mato Grosso do Sul, Campus de \\ Navirai, Navirai, MS, Brasil. \\ ${ }^{2}$ Departamento de Ciências Exatas, Faculdade de Ciências Agrárias e Veterinárias, Universidade \\ Estadual Paulista, Jaboticabal, SP, Brasil.
}

Recebido em: 28 de Agosto de 2018 - Aceito em: 4 de Abril de 2019

\begin{abstract}
Resumo
A utilização de dados provenientes de modelos atmosféricos globais é uma alternativa para dados meteorológicos de superfície faltantes e sem séries históricas homogêneas subsidiando avaliações em áreas sem informações meteorológicas de superfície bem como na modelagem do balanço hídrico. Assim, objetivou-se determinar a acurácia da reanálise ERA-Interim do ECMWF e avaliar sua aplicação na estimativa da deficiência hídrica no estado do Paraná, Brasil. Foram utilizados dados meteorológicos decendiais de temperatura média do ar $\left({ }^{\circ} \mathrm{C}\right)$ e precipitação pluviométrica $(\mathrm{mm})$ do período de 1989 a 2014 oriundos do ERA-Interim do ECMWF e de dados de superfícies (INMET). Para uma avaliação mais criteriosa, os dados meteorológicos foram estratificados por macrorregiões e estações do ano. Foi calculado o balanço hídrico climatológico segundo Thorthwaite e Mather (1955) para ambas as fontes de dados com CAD = $100 \mathrm{~mm}$. Os mapas foram gerados usando sistema de informação geográfica (SIG) e as comparações entre o ERAInterim do ECMWF e os dados das estações meteorológicas foram realizados pelos índices que avaliam a acurácia, precisão e tendência dos dados. $\mathrm{A}_{\mathrm{AIR}}$ do ERA-Interim do ECMWF demostrou alta acurácia em todas as estações do ano, com MAPE $>5 \%$ e desvios menores que $<2{ }^{\circ} \mathrm{C}$ para todo Estado do Paraná. O teste Tukey comprovou a alta acurácia, pois evidenciou igualdade entre os dados de $\mathrm{T}_{\text {ERA-I }}$ e $\mathrm{T}_{\text {INMET. }}$ A $\mathrm{P}_{\text {ERA-I }}$ subestimou por todo momento a $\mathrm{P}_{\text {INMET }}$ que ocorreu nas macrorregiões Noroeste, Norte e Sudoeste, evidenciando baixas precisões $\left(\mathrm{R}^{2} \cong 0\right)$ e elevados MAPE (> 15\%). O teste de Tukey comprovou que a $\mathrm{P}_{\text {ERA-I }}$ e $\mathrm{P}_{\text {INMET }}$ são diferentes, e assim, demonstra que a $\mathrm{P}_{\text {ERA-I }}$ necessita de correções para ser utilizadas. A acurácia do $\mathrm{DEF}_{\text {ERA-I }}$ foi razoável demonstrando um índice de confiança de $\mathrm{C}=0,69$, a qual corresponde a um desempenho "bom".
\end{abstract}

Palavras-chave: balanço hídrico, big data, modelagem climática, precipitação, temperatura do ar.

\section{Accuracy of ECMWF ERA-Interim Reanalysis and its Application in the Estimation of the Water Deficieny in Paraná, Brazil}

\begin{abstract}
The use of data from global atmospheric models is an alternative to missing surface meteorological data, but few studies have determined the accuracy of these models and evaluated their use in water balance modeling. We determine the accuracy of the ECMWF ERA-Interim reanalysis dataset and its application in the calculations of water deficiency in Paraná, Brazil. We used 10-day period mean air temperature $\left({ }^{\circ} \mathrm{C}\right)$ and rainfall $(\mathrm{mm})$ monthly data from 1989 to 2014 from ECMWF ERA-Interim and surface data (INMET). For a more careful evaluation, the meteorological data were stratified by macro regions and seasons. We calculated the climatological water balance according to Thorthwaite and Mather (1955) for both data sources with $\mathrm{CAD}=100 \mathrm{~mm}$. The maps were generated using geographic information sys-
\end{abstract}

Send correspondence to Lucas Eduardo de Oliveira Aparecido, Lucas-aparecido@outlook.com. 
tem (SIG) and the comparisons between the ECMWF ERA-Interim data and the meteorological station data were performed by the indexes that evaluate the accuracy, precision and trend of the data. The $\mathrm{T}_{\mathrm{AIR}}$ of ECMWF ERA-Interim showed high accuracy in all seasons of the year, with MAPE $>5 \%$ and lower than $<2^{\circ} \mathrm{C}$ for all Paraná State. The Tukey test proved the high accuracy, as it evidenced by the equality between the $\mathrm{T}_{\text {ERA-I }}$ and $\mathrm{T}_{\text {INMET }}$ data. The $\mathrm{P}_{\text {ERA-I }}$ consistently underestimated the $\mathrm{P}_{\text {INMETs }}$ that occurred in the Northwest, North and Southwest macro-regions, showing low accuracies $(\mathrm{R} 2 \cong 0)$ and high MAPE $(>15 \%)$. The Tukey test proved that the $\mathrm{P}_{\text {ERA-I }}$ and $\mathrm{P}_{\text {INMET }}$ are different, and thus demonstrates that the $\mathrm{P}_{\text {ERA-I }}$ needs corrections to be used. The accuracy of the DEF $\mathrm{ERA}_{\text {-I }}$ was reasonable demonstrating a confidence index of $\mathrm{C}=0.69$, which corresponds to a "good" performance.

Keywords: water balance, bigdata, climate modeling, rainfall, air temperature.

\section{Introdução}

O Paraná tem a quinta maior economia do Brasil e tem como um dos principais setores produtivos o agrícola (Rodrigues, Moretto, Guilhoto, 2015), produzindo feijão, café, algodão, trigo, mandioca e cana-de-açúcar (Aparecido et al. 2016). Assim como no território brasileiro, não dispõe de uma rede de estações meteorológicas de superfície que atenda todas as necessidades agrícolas. Um dos principais problemas são as grandes falhas nos dados nas séries temporais (Pereira et al. 2002), o que dificulta a utilização dos dados nas tomadas de decisões dos produtores.

As variáveis climáticas quando aferidas corretamente, se tornam excelentes ferramentas estratégicas (Niu et al. 2008), podendo ser empregadas desde modelos de simulação de crescimento (Gouranga e Ashwani, 2014) até na elaboração de cartas de zoneamento agrícola (Rahimi et al. 2013). No entanto, as falhas nas séries de dados acabam limitando diversas pesquisas de monitoramento dos cultivos, previsões de produtividade, pois, além de ter o conhecimento das condições vegetativas é necessário ter dados meteorológicos e confiáveis. $\mathrm{O}$ monitoramento das condições climáticas é afetado por diversos problemas como, por exemplo, a perda de calibração dos sensores, a baixa densidade de estações, as falhas de medidas e a falta de continuidade da série histórica, além do fechamento de estações (Moraes et al. 2012).

Uma alternativa é o uso de dados de modelos atmosféricos globais como o European Center for MediumRange Weather Forecast (ECMWF). Este modelo é utilizado em um sistema de assimilação de dados que coleta informações de diversas fontes meteorológicas no mundo, como por exemplo, os radares meteorológicos e satélites (ECMWF, 2009; Couto et al. 2015). O sistema ECMWF fornece facilmente os dados para download pelo banco meteorológico do JRC (Joint Research Centre), um centro de pesquisas da Comissão Europeia (Person e Grazziani, 2007; Moraes; Rocha; Lamparelli, 2014), Incluído informações de chuva $(\mathrm{mm})$, temperaturas do $\operatorname{ar}\left({ }^{\circ} \mathrm{C}\right)$, radiação solar $\left(\mathrm{MJ} \mathrm{m}^{-2}\right)$ e evapotranspiração $(\mathrm{mm})$ por PenmanMonteith-FAO.

Alguns trabalhos verificando a acurácia dos produtos de reanálise do ERA-40 foram encontrados na literatura, como Fu e Betts (2008), que avaliando a acurácia do ERA40 na região amazônica encontraram subestimação ou superestimação do ERA-40 em relação aos informados por estações meteorológicas de superfície, para as variáveis de chuva e temperatura do ar. Sapucci (2014) realizou o monitoramento da umidade no território brasileiro com dados do ERA-40, e observou que os valores da reanálise do ECMWF apresentaram uma maior dispersão dos dados. Contudo, não foram encontrados trabalhos para o Estado do Paraná que avaliasse a utilização de nenhum dos produtos de reanálise do ECMWF (ERA-15, ERA-40, ERAInterim ou ERA-5) na modelagem da deficiência hídrica (DEF).

A deficiência hídrica é a relação em milímetros da diferença entre a evapotranspiração potencial (ETP) e a real (ETR), assim, expressa a quanto que o cultivo agrícola deixou de evapotranspirar em função do baixo nível de armazenamento água no solo (Moreto et al. 2015; Aparecido et al. 2018). É uma variável meteorológica importante, pois tem relação direta com a produtividade e qualidade de diversos cultivos agrícolas (Martins et al. 2015; Battisti e Sentelhas, 2015).

A utilização de informações meteorológicas provenientes de produtos de reanálise do ECMWF é uma alternativa viável aos dados meteorológicos de superfície, mas poucas pesquisas determinaram sua acurácia ou avaliaram sua utilização na modelagem do balanço hídrico climatológico. Assim, objetivou-se determinar a acurácia e a precisão da reanálise ERA-Interim do ECMWF e avaliar sua aplicação na estimativa da deficiência hídrica(DEF).

\section{Material e Métodos}

Foram utilizados dados meteorológicos decendiais de temperatura média do ar $\left({ }^{\circ} \mathrm{C}\right)$ e precipitação pluviométrica $(\mathrm{mm})$ do período de 1989 a 2014 oriundos do conjunto de dados do ERA Interim, uma reanálise atmosférica global produzido pelo European Center for Medium-Range Weather Forecast (ECMWF) e também de estações meteorológicas de superfícies, ambos do Estado do Paraná, Brasil (Fig 1). O Paraná ocupa uma área de $199.315 \mathrm{~km}^{2}$ no Sul do Brasil e apresenta 4 classes climáticas (Köppen e Geiger, 1928): Aw, Cwa, Cfa, and Cfb. A classe Cfa é a mais predominante (Aparecido et al. 2016).

Os dados climáticos das estações de superfície foram obtidos de 399 estações meteorológicas oriundas do SIMEPAR e INMET (Fig. 1). Os dados providenciados do ERA-Interim foram obtidos em escala decendial, com uma resolução espacial de 0,25 graus $( \pm 25 \times 25 \mathrm{~km})$, corres- 
pondendo a 598 pontos de estações virtuais que contemplam o Estado do Paraná (Fig. 1). Para obter resultados mais criteriosos e regionais os dados meteorológicos foram estratificados por macrorregiões e separados por estações do ano obedecendo aos critérios da Tabela 1.

Para comparar a influência das fontes dos dados no cálculo da deficiência hídrica anual, foram calculados o balanço hídrico climatológico segundo Thorthwaite e Mather (1955) com capacidade de armazenamento igual a $100 \mathrm{~mm}$ para ambos os tipos de dados (INMET x ERAInterim). Por meio do sistema de informações geográficas (SIG) realizou a interpolação espacial do ERA-Interim e dos dados de superfícies de todo o estado do Paraná pelo o método de krigagem (Krige, 1951) usando modelo esférico com um vizinho. O sistema de projeção cartográfica utilizado na projeção foi a cônica de Lambert. Após o processo de interpolação dos dados, as malhas geradas foram sobrepostas para realizar a comparação do ERA-Interim com o INMET, sempre com as mesmas coordenadas geográficas.

A comparação entre o ERA-Interim e os dados das estações meteorológicas de superfície foi realizada pelos índices estatísticos: 1) correlação de Pearson (r);2) Coeficiente de determinação ajustado ( $\mathrm{R}^{2}$ adjusted); 3 ) Erro aleatório (Ea); 4) Erro sistemático (Es); 5) Erro absoluto
Tabela 1 - Conversão para o período de cada estação do ano.

\begin{tabular}{lc}
\hline Estações do ano & Período \\
\hline Verão & $21 / \mathrm{DEZ}+\mathrm{JAN}+\mathrm{FEV}+20 / \mathrm{MAR}$ \\
Outono & $21 / \mathrm{MAR}+\mathrm{ABR}+\mathrm{MAI}+20 / \mathrm{JUN}$ \\
Inverno & $21 / \mathrm{JUN}+\mathrm{JUL}+\mathrm{AGO}+23 / \mathrm{SET}$ \\
Primavera & $24 / \mathrm{SET}+\mathrm{OUT}+\mathrm{NOV}+20 / \mathrm{DEZ}$ \\
\hline
\end{tabular}

máximo (EAmax); 6) Erro quadrático médio (MSE); 7) Erro quadrático médio da raiz (RMSE); 8) Erro médio absoluto (MAE); 9) Erro percentual absoluto médio (MAPE) (Eqs. (1) a (9). O desvio foi calculado subtraindo o valor previsto pelo ERA-Interim dos dados reais de superfície (Xreal - Xera-I).

$$
\begin{gathered}
r=\frac{\sum_{i=1}^{n}\left(\text { Yobs }_{i}-\overline{Y_{\text {obs }}}\right) X\left(Y_{\text {est }}-Y_{\text {est }}\right)}{\sqrt{\sum_{i=1}^{n}\left(\text { Yobs }_{i}-\overline{Y_{\text {obs }}}\right)^{2}} X \sqrt{\sum_{i=1}^{n}\left(\text { Yest }_{i}-\overline{Y_{\text {est }}}\right)^{2}}} \\
R_{\text {adjusted }}^{2}=\left[1-\frac{\left(1-R^{2}\right) X(n-1)}{N-k-1}\right]
\end{gathered}
$$

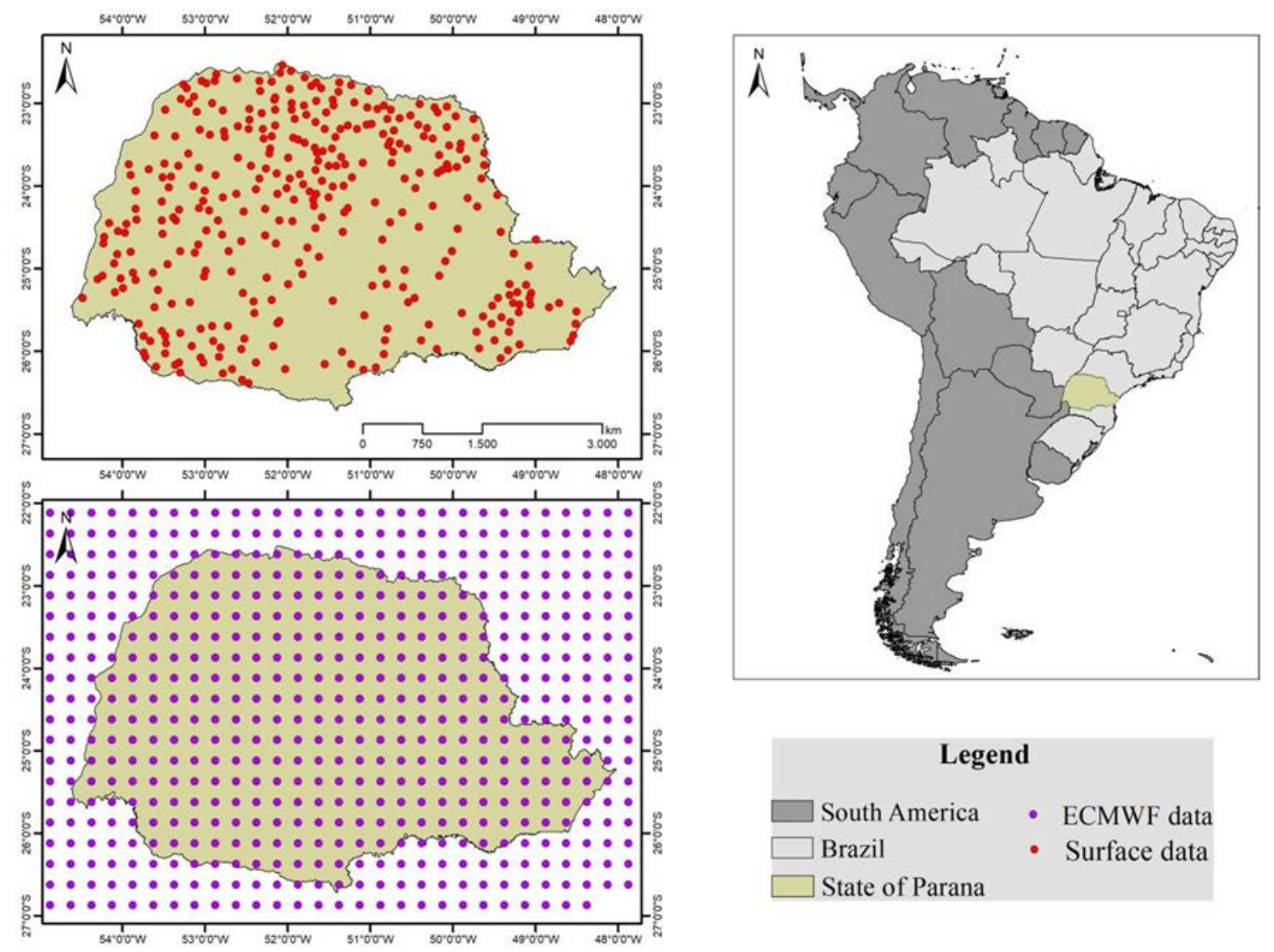

Figura 1 - Localização do Paraná e dados de superfície A) e estações virtuais do ECMWF B). 


$$
\begin{aligned}
& E a=\sqrt{\frac{\sum_{i=1}^{N}\left(Y_{E S T_{i}}-\bar{Y}\right)^{2}}{N}} \\
& E s=\sqrt{\frac{\sum_{i=1}^{n}\left(Y o b s_{i}-\bar{Y}\right)^{2}}{N}} \\
& E A_{\max }=\max \left(\left|Y_{o b s_{i}}-Y_{e s t_{i}}\right|\right)_{i=1}^{n} \\
& M S E=\frac{\sum_{i=1}^{N}\left(Y_{O B S_{i}}-Y_{E S T_{i}}\right)^{2}}{N} \\
& R M S E=\sqrt{\frac{\sum_{i=1}^{n}\left(Y_{o b s_{i}}-Y_{e s t_{i}}\right)^{2}}{N}} \\
& M A E=\frac{\sum_{i=1}^{N}\left|Y_{O B S_{i}}-Y_{E S T_{i}}\right|}{N} \\
& \operatorname{MAPE}(\%)=\frac{\sum_{i=1}^{N}\left(\left|\frac{\text { Yest }_{i}-\text { Yobs }_{i}}{\text { Yobs }_{i}}\right| \times 100\right)}{N}
\end{aligned}
$$

em que, $Y_{\text {est }}$ : variável interpolada; $Y_{o b s_{i}}$ : variável observada; $N$ : número de dados e $k$ : número de variáveis independentes na regressão.

O confronto entre os dados do INMET e ERA-Interim também foi realizada pelo Test $\mathrm{F}$ e quando houve significância, foi aplicado o Teste de comparação de médias de Tukey (95\% de confiabilidade).

\section{Resultados e Discussão}

Os dados de temperatura média do ar $\left(\mathrm{T}_{\mathrm{AIR}}\right)$ oriundos do ERA-Interim foram semelhantes espacialmente aos dados meteorológicos de superfície. As maiores $\mathrm{T}_{\mathrm{AIR}}$ ocorreram na região Noroeste e as menores na região Sul (Fig. 2). No verão, o ERA-Interim demonstrou desvios entre $-1,4$ a $0,9^{\circ} \mathrm{C}$ (Fig. 3.A), o que comprova uma elevada acurácia $(\mathrm{MAPE}=1,83 \%)$ e precisão $\left(\mathrm{R}^{2}\right.$ adj $\left.=0,94\right)$ para o Estado do Paraná (Fig. 4). Os dados do ERAInterim da região Sul demonstram as maiores acurácias, com MAPE de apenas 1,39\%. Um MAPE de 1,39\% é muito baixo, pois em uma determinada região onde predomina uma $\mathrm{T}_{\mathrm{AIR}}$ em torno de $30^{\circ} \mathrm{C}$, ocorre uma variação de apenas $\pm 0,38^{\circ} \mathrm{C}$.

No outono, a $\mathrm{T}_{\mathrm{AIR}}$ do ERA-Interim evidenciou desvios variando entre $-1,7$ e $0,6^{\circ} \mathrm{C}$ (Fig. 3.C). A acurácia e a precisão das previsões pelo ERA-Interim foram de 2,72\% (MAPE) e 0,94 ( ${ }^{2}$ adj) para todo Estado (Fig. 5.E). Ponderando por macrorregiões, o ERA-Interim evidenciou maiores acurácia na macrorregião Norte $(\mathrm{MAPE}=2,16 \%)$
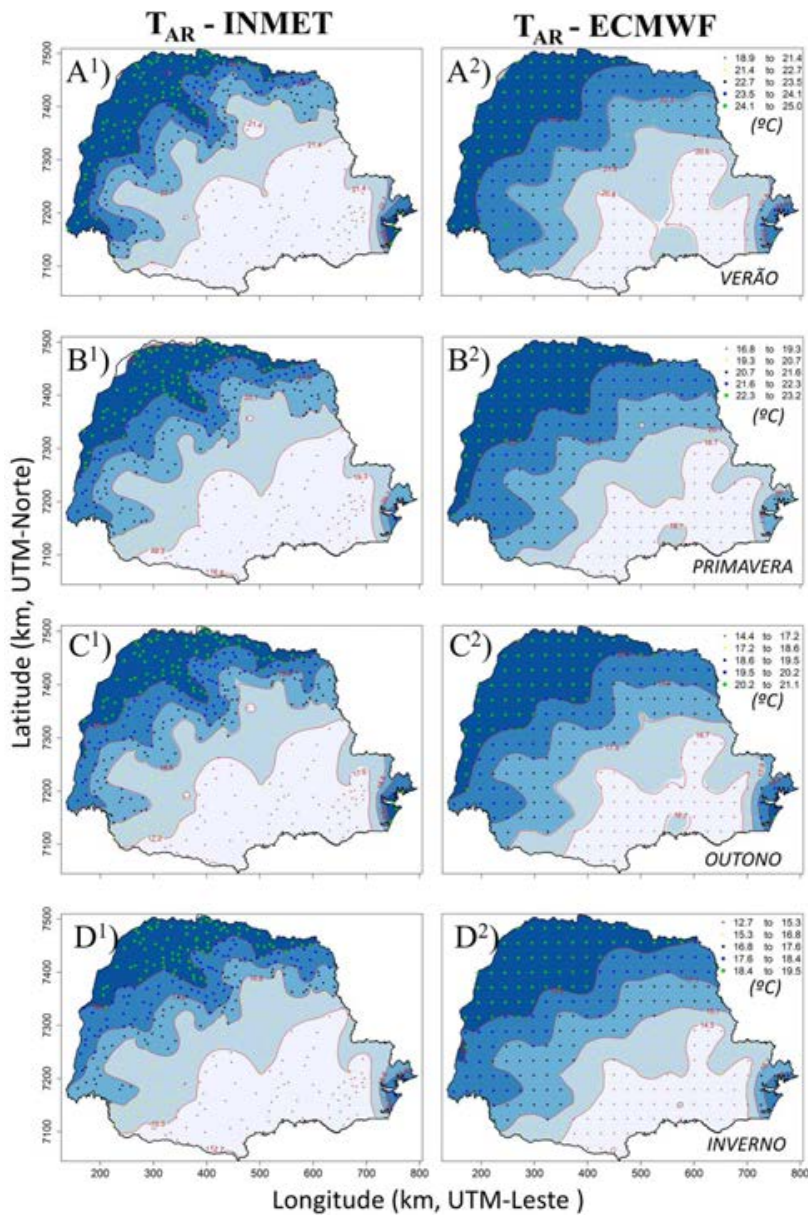

Figura 2 - Temperatura do ar média dos dados de superfície (A) e do ECMWF (B) para o Estado do Paraná. Valores negativos na legenda indicam subestimativas feitas pelo ECMWF.

e os menores na macrorregião Noroeste (MAPE $=4,09 \%$ ), na qual o ERA-Interim subestimou os dados do INMET por todo período (Fig. 5.A).

Durante o inverno, o ERA-Interim demonstrou uma espacialização similar aos dados de superfície do INMET, evidenciado uma redução dos valores de $\mathrm{T}_{\mathrm{AIR}}$ no sentido Noroeste - Sudoeste. Com a sobreposição dos mapas, os desvios entre o ERA-Interim e os dados de superfície variaram de $-1,3$ a $1,0{ }^{\circ} \mathrm{C}$, que gerou MAPEs de 3,46; 2,26; 1,92 e $2,23 \%$ para as regiões Noroeste, Norte, Sudoeste e Sul, respectivamente (Fig. 6).

Na primavera, a $\mathrm{T}_{\mathrm{AIR}}$ do ERA-Interim foi similar os dados de superfície (Fig. 2) com desvios variando entre $-1,6$ a $-1,1^{\circ} \mathrm{C}$. Os maiores desvios ocorreram na macrorregião Noroeste, na qual se predomina também as mais elevadas $\mathrm{T}_{\mathrm{AIR}}$ do Estado do Paraná (Ricce et al. 2014), evidenciando que o ERA-Interim subestima nas regiões onde ocorrem as mais altas $\mathrm{T}_{\mathrm{AIR}}$. $\mathrm{A}$ acurácia da $\mathrm{T}_{\mathrm{AIR}}$ na primavera foi de MAPE $=3,04 \%$ e $\mathrm{R}^{2}$ adj $=0,96$ para todo Estado do Paraná (Fig. 7). Em relação às macrorregiões do Estado, ERA-Interim subestimou em todo período nas 

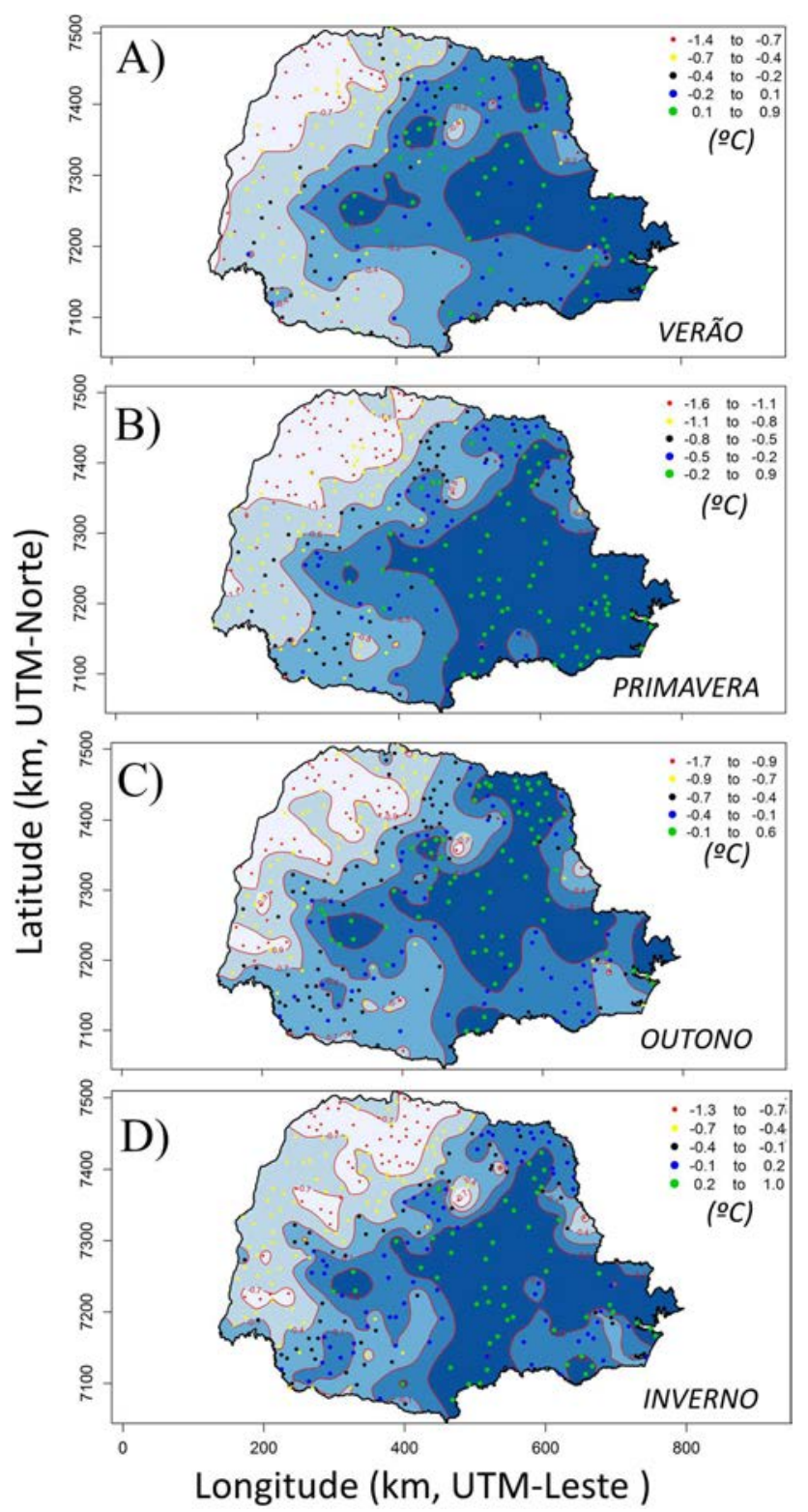

Figura 3 - Desvios entre as temperaturas do ar média dos dados de superfície e do ECMWF para as estações do verão (A), primavera (B), outono (C) e inverno (D) para o Estado do Paraná. Valores negativos na legenda indicam subestimativas feitas pelo ECMWF.

macrorregiões Noroeste e Sudoeste, evidenciando os maiores MAPEs, sendo 4,64 e 3,41\%, respectivamente (Fig. 7. A.C). Por sua vez, a macrorregião em que o ERAInterim demonstrou os menores erros foi na Sul com MAPE de $1,58 \%$.

A precipitação pluviométrica do ERA-Interim apresentou maiores diferenças aos dados de superfície do INMET (Fig. 8). O P que ocorreu no verão do Estado do Paraná variou de 476,6 a $638,1 \mathrm{~mm}$, e o ERA-Interim demonstrou baixa similaridade na distribuição dos dados, evidenciando desvios que variaram de $-145,3$ até $226,5 \mathrm{~mm}$, para as macrorregiões Noroeste e Sudoeste,
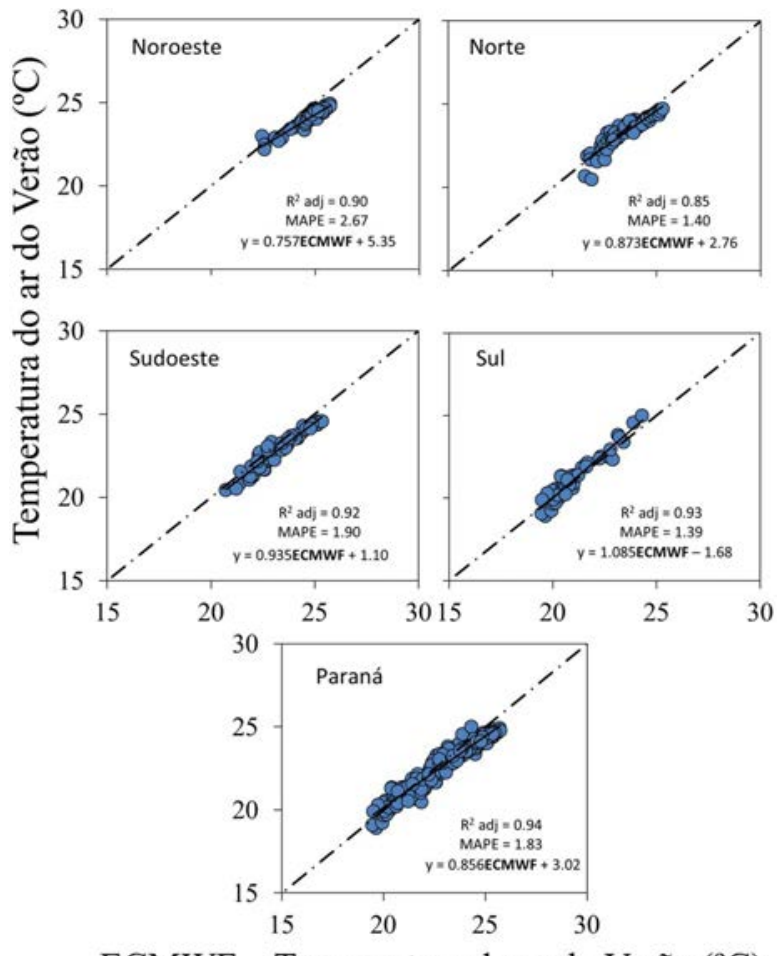

ECMWF - Temperatura do ar do Verão $\left({ }^{\circ} \mathrm{C}\right)$

Figura 4 - Temperatura média do ar $\left({ }^{\circ} \mathrm{C}\right)$ do ECMWF em função dos dados meteorológicos de superfície para a estação do verão nas macrorregiões do Estado do Paraná, Brasil.

respectivamente (Fig. 9.A). Os dados do $\mathrm{P}_{\text {ERA-I }}$ subestimaram as $\mathrm{P}$ que ocorreram nas macrorregiões Noroeste, Norte e Sudoeste, evidenciando baixas precisões $\left(\mathrm{R}^{2}=0\right)$ e elevados MAPE (> 15\%).

O ERA-Interim não acompanhou a tendência espacial dos dados de $\mathrm{P}$ de superfície no verão. Na macrorregião Sul o ERA-Interim subestimou quando houve precipitações maiores que $530 \mathrm{~mm}$ (Fig. 10), evidenciando que não importa a região, se houver elevadas precipitações, os dados de reanálise ERA-Interim apresentam dificuldades na previsão. Moraes et al. (2012) também observou que a reanálise do ERA-Interim apresenta acurácia moderada na previsão da Precipitação.

$\mathrm{Na}$ estação do outono, os dados de $\mathrm{P}_{\text {ERA-I }}$ tiveram maiores similaridades, acompanhando parte da distribuição dos dados de superfície, evidenciando desvios de apenas $12,1 \mathrm{~mm}$ em algumas áreas das macrorregiões Sudoeste e Sul e desvios maiores de 158,9 mm nas macrorregiões Noroeste, Norte e no Litoral. A variação de $12,1 \mathrm{~mm}$, como os que ocorreram nas localidades de Pato Branco, União da Vitoria e Palmas, são considerados baixos para um período de 3 meses (estação do ano), pois equivale a um erro aproximadamente $4 \mathrm{~mm}$ mensal.

No outono, o ERA-Interim superestimou as P que ocorreram em todas as macrorregiões do Estado Paraná. Os dados de reanálise do ERA-Interim demostraram 


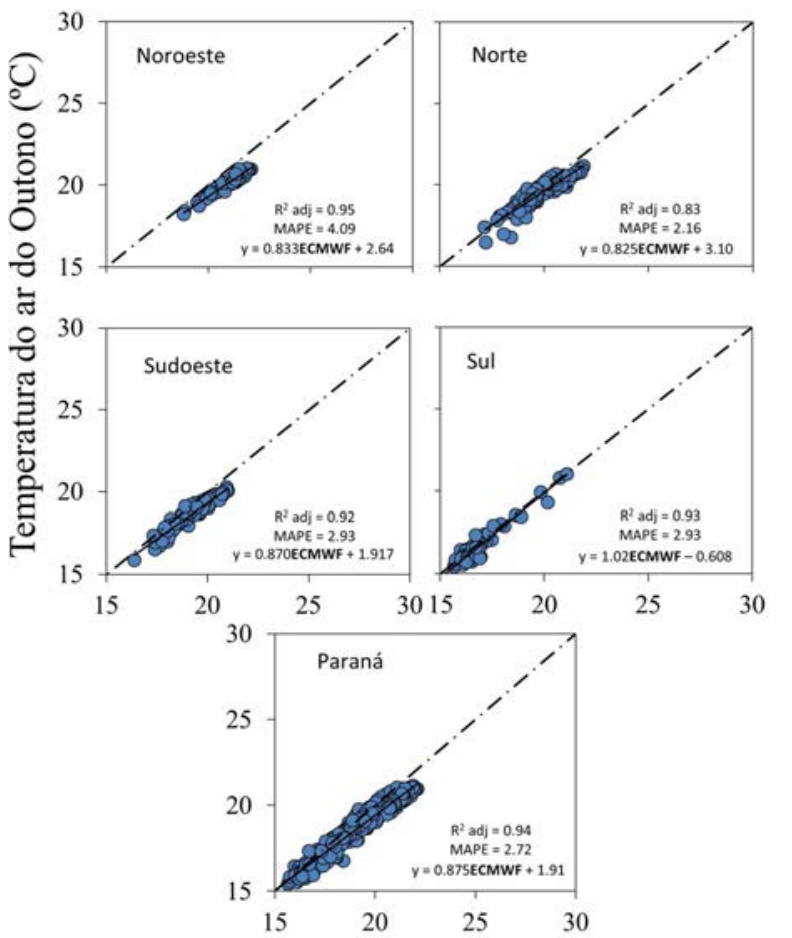

ECMWF - Temperatura do ar do Outono $\left({ }^{\circ} \mathrm{C}\right)$

Figura 5 - Temperatura média do ar $\left({ }^{\circ} \mathrm{C}\right)$ do ECMWF em função dos dados meteorológicos de superfície para a estação do outono nas macrorregiões do Estado do Paraná, Brasil.
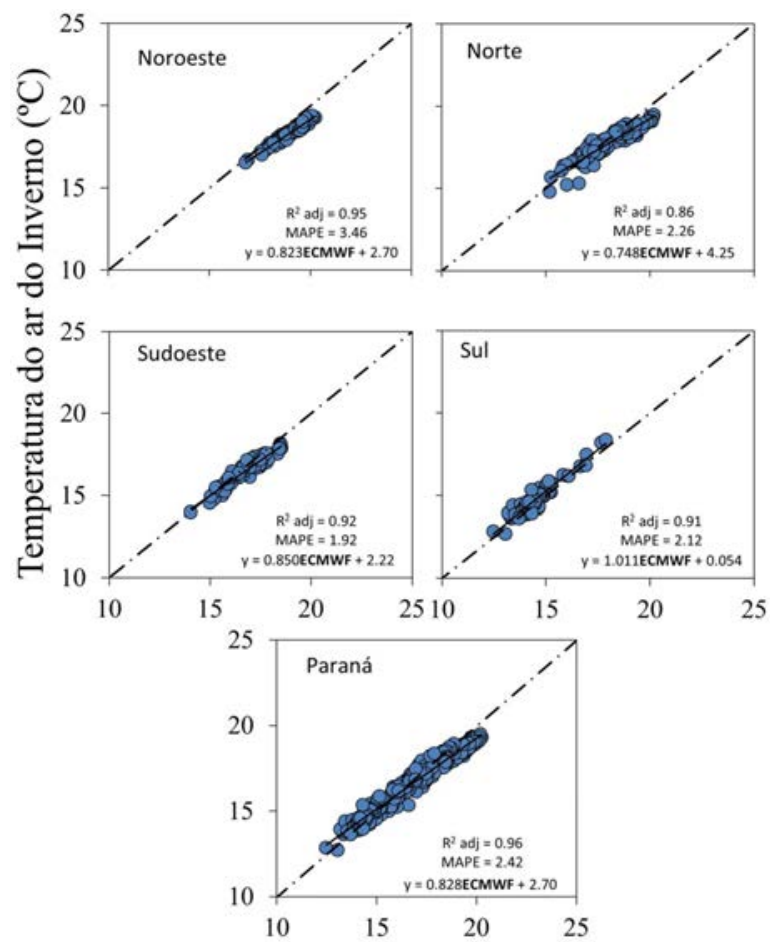

ECMWF - Temperatura do ar do Inverno $\left({ }^{\circ} \mathrm{C}\right)$

Figura 6 - Temperatura média do ar $\left({ }^{\circ} \mathrm{C}\right)$ do ECMWF em função dos dados meteorológicos de superfície para a estação do inverno nas macrorregiões do Estado do Paraná, Brasil.
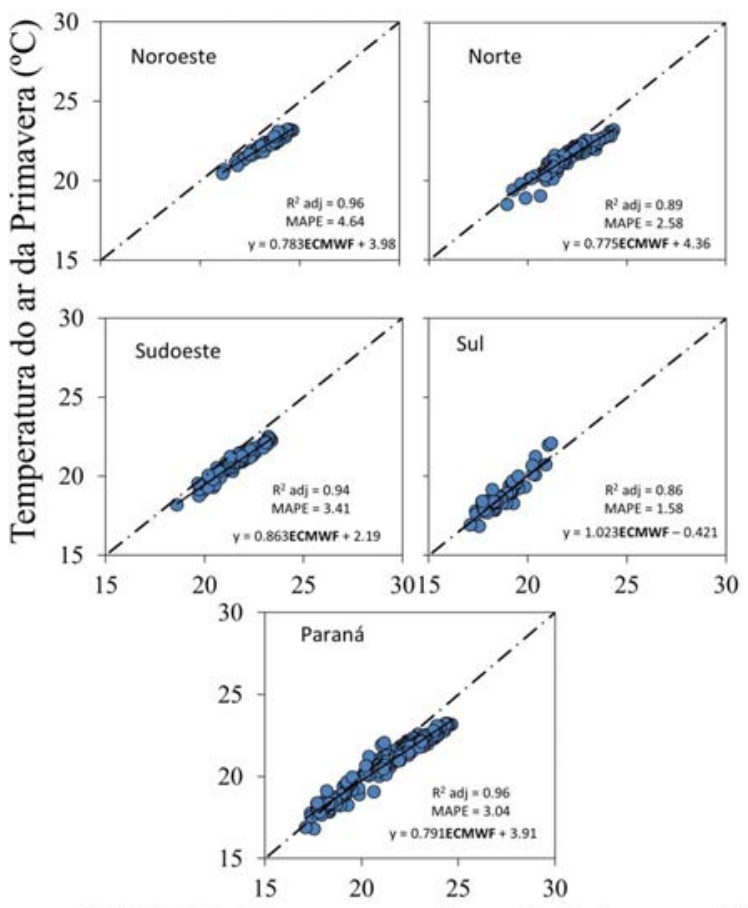

ECMWF - Temperatura do ar da Primavera $\left({ }^{\circ} \mathrm{C}\right)$

Figura 7 - Temperatura média do ar $\left({ }^{\circ} \mathrm{C}\right)$ do ECMWF em função dos dados meteorológicos de superfície para a estação da primavera nas macrorregiões do Estado do Paraná, Brasil.

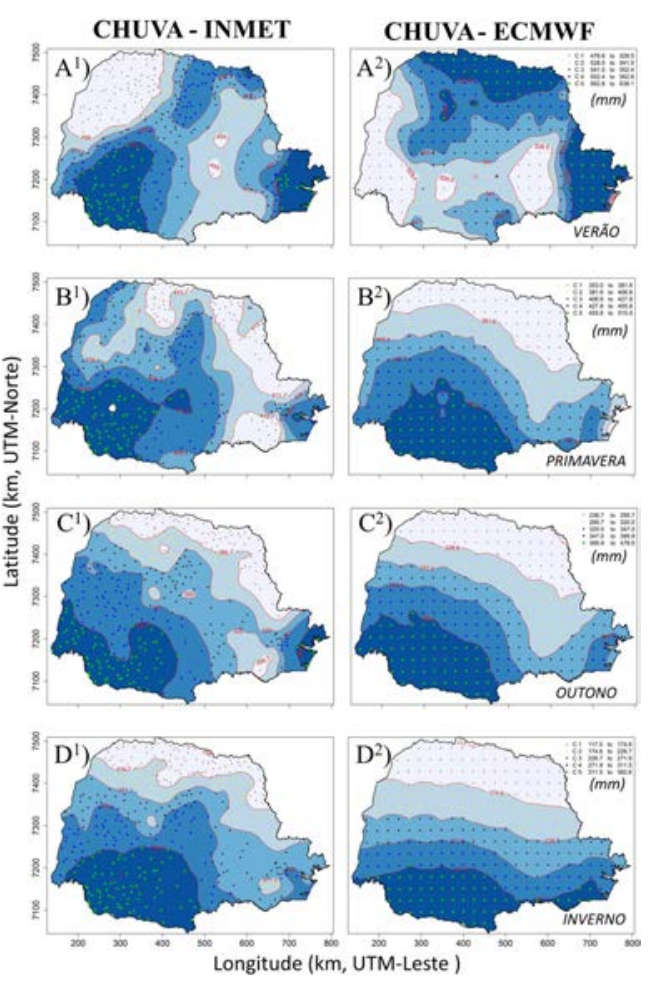

Figura 8 - Precipitação média dos dados de superfície (A) e do ECMWF (B) para o Estado do Paraná. Valores negativos na legenda indicam subestimativas feitas pelo ECMWF. 

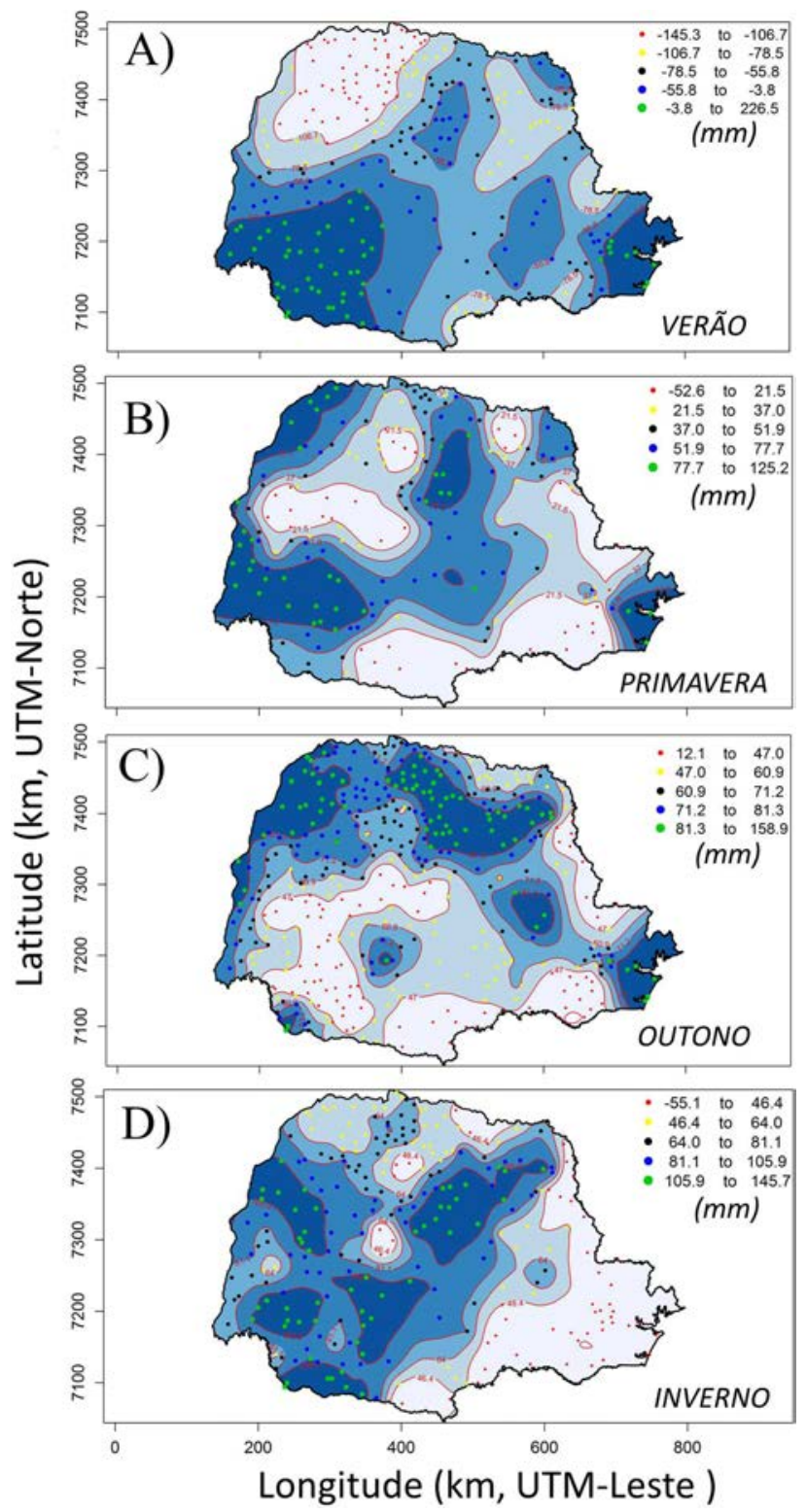

Figura 9 - Desvios entre as precipitações dos dados de superfície e do ECMWF para as estações do verão (A), primavera (B), outono (C) e inverno (D) para o Estado do Paraná. Valores negativos na legenda indicam subestimativas feitas pelo ECMWF.

valores médios de precisão, com $\mathrm{R}^{2}$ adj variando de $0,52 \mathrm{a}$ 0,89 para as macrorregiões Sul e Noroeste, respectivamente (Fig. 11.A a D), demonstrando no outono tendências semelhantes aos dados de superfície.

A P na estação do inverno variou de 117 a 392 mm, evidenciando uma redução gradual da precipitação no sentido Norte-Sul, sendo que os níveis mais baixos de $\mathrm{P}$ ocorreram nas macrorregiões Noroeste e Norte, e os mais elevados na macrorregião Sudoeste. Os dados do $\mathrm{P}_{\text {ERA-I }}$ evidenciaram desvios entre $-55,1$ a $145,7 \mathrm{~mm}$ e superestimação nas macrorregiões Nordeste, Norte e Sudoeste, evidenciando valores de $\mathrm{R}^{2}$ adj de 0,$87 ; 0,67$ e 0,85 respec-

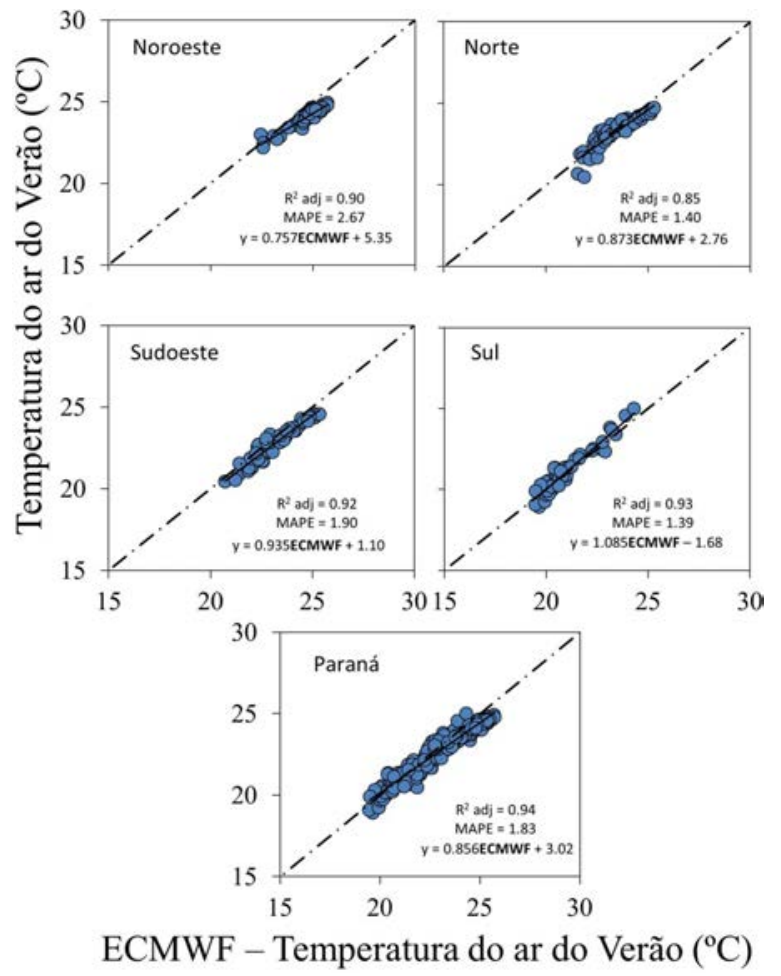

Figura 10 - Precipitação pluviométrica do ECMWF em função dos dados meteorológicos de superfície para a estação do verão nas macrorregiões do Estado do Paraná, Brasil.

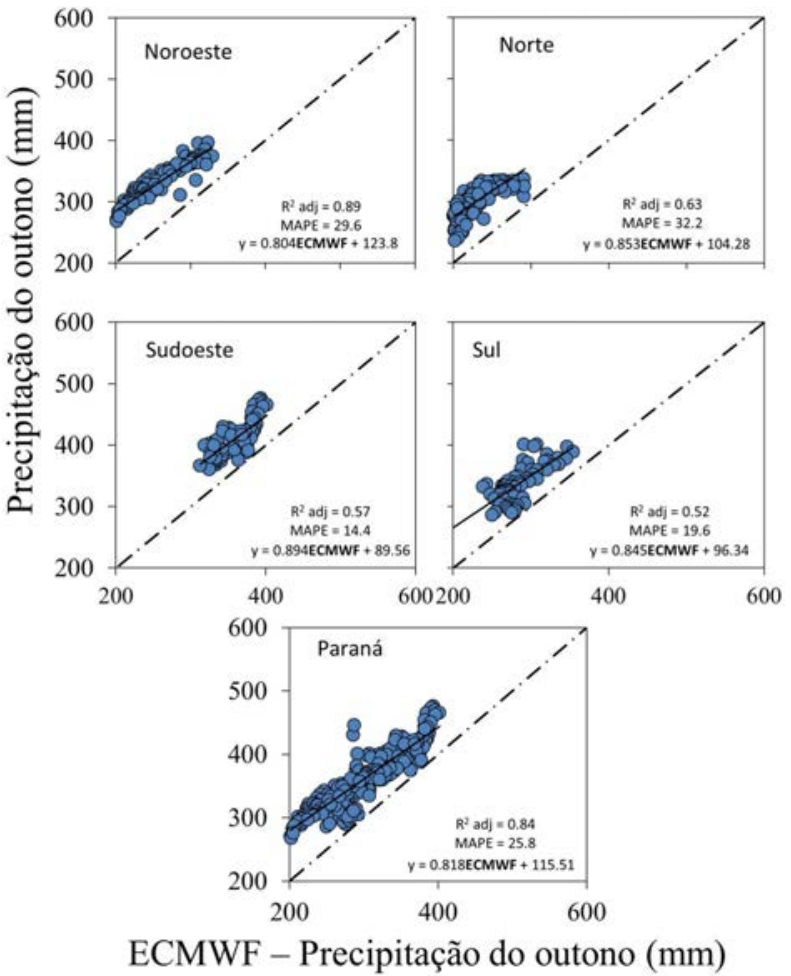

Figura 11 - Precipitação pluviométrica do ECMWF em função dos dados meteorológicos de superfície para a estação do outono nas macrorregiões do Estado do Paraná, Brasil. 
tivamente (Fig. 12). Na macrorregião Sul a superestimação do ERA-Interim ocorre com P abaixo de $370 \mathrm{~mm}$.

$\mathrm{Na}$ estação da primavera, a $\mathrm{P}_{\text {ERA-I }}$ não acompanhou a tendência e distribuição dos dados de superfície do Estado do Paraná, demonstrando a ocorrência de desvios que variaram de -52 até $125,2 \mathrm{~mm}^{\text {estação }}{ }^{-1}$ (Fig. 9). Os dados do ERA-Interim demonstraram valores baixos de precisão, com $\mathrm{R}^{2}$ adj variando de 0,17 a 0,57 para as macrorregiões Noroeste e Norte, respectivamente. O menor MAPE ocorreu na macrorregião Noroeste, sendo o valor de $9,48 \%$ (Fig. 13).

Uma síntese da acurácia dos dados de $\mathrm{T}_{\mathrm{AIR}}$ e $\mathrm{P}$ oriundos do ERA-Interim para todas as regiões do Paraná e estações do ano podem ser observadas na Tabela 2. A $\mathrm{T}_{\mathrm{AIR}}$ demostra claramente a alta acurácia em todas as estações do ano, com MAPE $>5 \%$ e desvios menores que $<2{ }^{\circ} \mathrm{C}$ (Fig. 14.A). Por exemplo, o MAPE da $\mathrm{T}_{\mathrm{AIR}}$ do inverno para a região Sul foi de $2,07 \%$, o que proporciona uma variação de apenas $\pm 0,46^{\circ} \mathrm{C}$ para uma $\mathrm{T}_{\mathrm{AIR}}$ média de $23,5^{\circ} \mathrm{C}$. Para a $\mathrm{P}$ oriunda do ERA-Interim a acurácia foi razoável, pois o MAPE chegou até 30,93\% como ocorreu na região Norte para a $\mathrm{P}_{\text {WINTER }}$, o que proporcionou desvios de até $180 \mathrm{~mm}$ (Fig. 14.B).

$\mathrm{O}$ teste de Tukey demostrou que a $\mathrm{T}_{\mathrm{AIR}}$ oriunda do ERA-Interim não tem diferenças significativas $(\mathrm{p}<0,05)$ em relação os dados do INMET (Fig. 15), o que já vem sendo evidenciado pelos autores Betts (1993), Durand

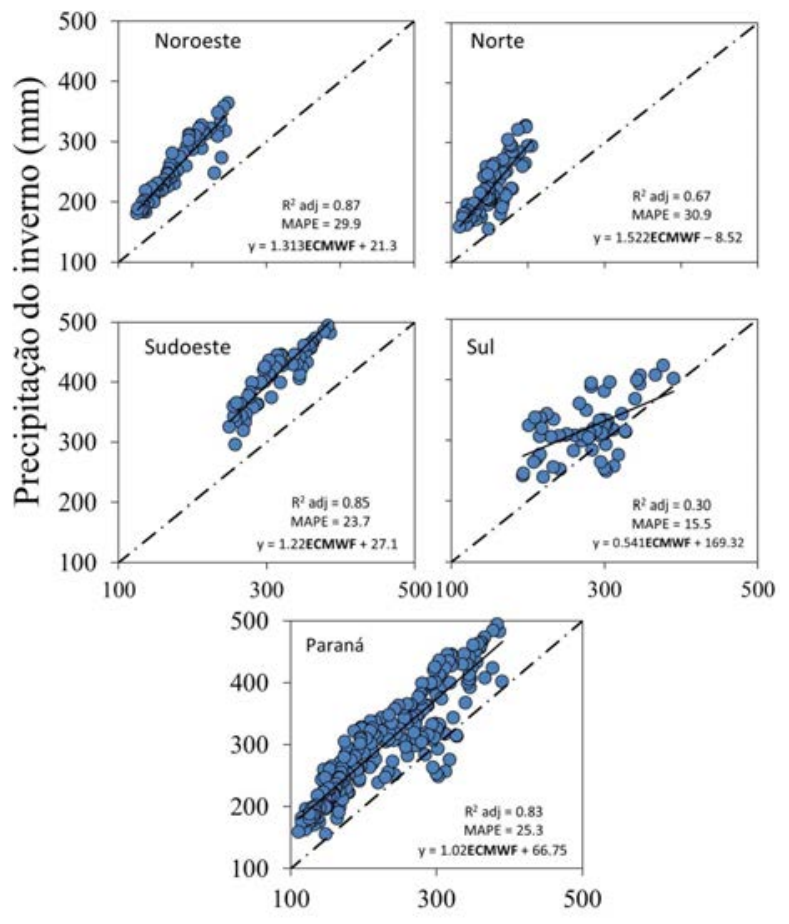

ECMWF - Precipitação do inverno (mm)

Figura 12 - Precipitação pluviométrica do ECMWF em função dos dados meteorológicos de superfície para a estação do inverno nas macrorregiões do Estado do Paraná, Brasil. et al. (2009) e Moraes et al. (2018). Entretanto, a P oriunda do ERA-Interim evidenciou ser diferente dos dados do INMET em todas as regiões do Paraná. Isso demonstra que a P precisa ser corrigida para ser repassada aos produtores, independente da estação do ano ou da região do Paraná.

Os maiores índices de deficiência hídrica (DEF) predominaram no Norte do Estado do Paraná, com valores máximos de $-240 \mathrm{~mm} \mathrm{ano}^{-1}$ (Fig. 16), valores esses considerados prejudiciais para vários cultivos agrícolas (Martins et al. 2015; Aparecido et al. 2017). Os menores DEF foram de $-30 \mathrm{~mm}$ e ocorreram na região do Sul onde estão as localidades de Pato Branco, Cascavel e Dois Vizinhos. A DEF estimada pelos dados do ERA-Interim acompanhou a tendência de distribuição espacial da DEF oriunda dos dados do INMET, mas houve superestimações de até $-92 \mathrm{~mm}$ ano $^{-1}$ na região Noroeste e subestimações de $148 \mathrm{~mm}^{-1}$ ano $^{-1}$ (Fig. 16.C).

A performance dos dados do $\mathrm{DEF}_{\text {ERA-I }}$ pode ser observada na Fig.17.A. A acurácia do DEF ERA-I $_{\text {foi }}$ razoável demonstrando os seguintes índices estatísticos: $\mathrm{r}=0,80 ; \mathrm{R}^{2}{ }_{\text {adj }}=0,64 ; \mathrm{Ea}=29,36 ;$ Es $=25,21$; EAmax $=137,67 ;$ MSE $=1497,57 ;$ RMSE $=38,70$; MAE $=32,39$ e MAPE $=47,13 \%$. O teste de Tukey ( $95 \%$ de confiabilidade) evidência que não existem diferenças significativas entre o DEF ERA-I $_{\text {I }}$ e DEF INMET (Fig. 17.B).

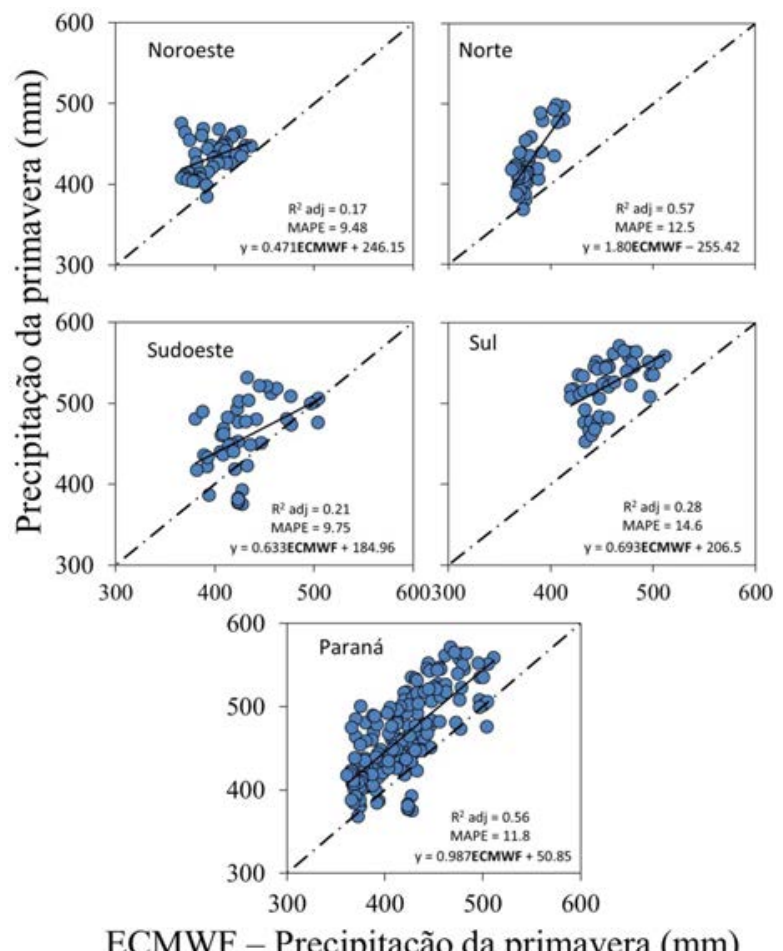

Figura 13 - Precipitação pluviométrica do ECMWF em função dos dados meteorológicos de superfície para a estação da primavera nas macrorregiões do Estado do Paraná, Brasil. 
Tabela 2 - Índices estatísticos para as temperaturas do ar e precipitações pluviométricas dos dados meteorológicos de superfície (INMET) e do ERAInterim para as estações do ano, em função das macrorregiões do Estado do Paraná, Brasil

\begin{tabular}{|c|c|c|c|c|c|c|c|c|c|c|}
\hline \multirow[t]{2}{*}{ Índices } & \multicolumn{5}{|c|}{ Temperatura do ar do verão } & \multicolumn{5}{|c|}{ Temperatura do ar da primavera } \\
\hline & Norte & Sul & Noroeste & Sudoeste & Estado & Norte & Sul & Noroeste & Sudoeste & Estado \\
\hline $\mathrm{r}$ & 0,92 & 0,96 & 0,95 & 0,96 & 0,97 & 0,94 & 0,92 & 0,98 & 0,97 & 0,98 \\
\hline $\mathrm{R}^{2}{ }_{\text {adj }}$ & 0,85 & 0,93 & 0,91 & 0,91 & 0,95 & 0,89 & 0,85 & 0,95 & 0,93 & 0,96 \\
\hline $\mathrm{Ea}$ & 0,37 & 0,30 & 0,22 & 0,30 & 0,39 & 0,38 & 0,35 & 0,16 & 0,27 & 0,40 \\
\hline Es & 0,19 & 0,21 & 0,66 & 0,39 & 0,32 & 0,58 & 0,17 & 1,10 & 0,74 & 0,68 \\
\hline EAmax & 1,40 & 0,93 & 1,15 & 0,93 & 1,40 & 1,58 & 1,04 & 1,54 & 1,20 & 1,58 \\
\hline MSE & 0,17 & 0,13 & 0,48 & 0,25 & 0,26 & 0,47 & 0,15 & 1,24 & 0,62 & 0,62 \\
\hline RMSE & 0,41 & 0,37 & 0,70 & 0,50 & 0,51 & 0,69 & 0,39 & 1,11 & 0,79 & 0,79 \\
\hline MAE & 0,33 & 0,29 & 0,67 & 0,44 & 0,43 & 0,58 & 0,30 & 1,09 & 0,74 & 0,68 \\
\hline \multirow[t]{3}{*}{ MAPE* } & 1,43 & 1,38 & 2,76 & 1,95 & 1,87 & 2,69 & 1,58 & 4,88 & 3,56 & 3,17 \\
\hline & \multicolumn{5}{|c|}{ Temperatura do ar do outono } & \multicolumn{5}{|c|}{ Temperatura do ar do inverno } \\
\hline & Norte & Sul & Noroeste & Sudoeste & Estado & Norte & Sul & Noroeste & Sudoeste & Estado \\
\hline $\mathrm{r}$ & 0,91 & 0,96 & 0,97 & 0,96 & 0,97 & 0,92 & 0,96 & 0,97 & 0,96 & 0,98 \\
\hline $\mathrm{R}^{2}$ adj & 0,82 & 0,92 & 0,94 & 0,92 & 0,95 & 0,85 & 0,92 & 0,95 & 0,93 & 0,96 \\
\hline $\mathrm{Ea}$ & 0,43 & 0,31 & 0,17 & 0,30 & 0,39 & 0,43 & 0,29 & 0,17 & 0,28 & 0,37 \\
\hline Es & 0,34 & 0,25 & 0,87 & 0,57 & 0,50 & 0,28 & 0,25 & 0,67 & 0,28 & 0,36 \\
\hline EAmax & 1,67 & 0,98 & 1,27 & 1,15 & 1,67 & 1,28 & 1,01 & 1,04 & 0,85 & 1,28 \\
\hline MSE & 0,30 & 0,16 & 0,78 & 0,42 & 0,41 & 0,27 & 0,14 & 0,48 & 0,16 & 0,26 \\
\hline RMSE & 0,55 & 0,40 & 0,88 & 0,65 & 0,64 & 0,52 & 0,38 & 0,69 & 0,40 & 0,51 \\
\hline MAE & 0,43 & 0,31 & 0,86 & 0,58 & 0,54 & 0,41 & 0,31 & 0,66 & 0,33 & 0,43 \\
\hline MAPE* & 2,24 & 1,90 & 4,28 & 3,14 & 2,83 & 2,33 & 2,07 & 3,60 & 1,97 & 2,48 \\
\hline \multirow[t]{2}{*}{ Índices } & \multicolumn{5}{|c|}{ Precipitação do verão } & \multicolumn{5}{|c|}{ Precipitação da primavera } \\
\hline & Norte & Sul & Noroeste & Sudoeste & Estado & Norte & Sul & Noroeste & Sudoeste & Estado \\
\hline r & 0,05 & 0,02 & 0,18 & 0,50 & 0,05 & 0,75 & 0,53 & 0,41 & 0,46 & 0,74 \\
\hline $\mathrm{R}^{2}{ }_{\text {adj }}$ & 0,00 & 0,00 & 0,03 & 0,25 & 0,00 & 0,56 & 0,28 & 0,17 & 0,21 & 0,55 \\
\hline $\mathrm{Ea}$ & 15,29 & 15,64 & 11,51 & 28,95 & 22,73 & 8,82 & 20,50 & 17,79 & 28,34 & 25,56 \\
\hline Es & 107,21 & 31,18 & 80,88 & 72,56 & 78,42 & 52,56 & 69,11 & 39,48 & 39,90 & 50,70 \\
\hline EAmax & 145,27 & 84,77 & 128,43 & 226,49 & 226,49 & 98,39 & 107,97 & 108,77 & 101,35 & 125,17 \\
\hline MSE & 11727,3 & 1216,8 & 6674,4 & 6102,9 & 6665,5 & 2839,8 & 5195,9 & 1874,7 & 2394,6 & 3223,7 \\
\hline RMSE & 108,29 & 34,88 & 81,70 & 78,12 & 81,64 & 53,29 & 72,08 & 43,30 & 48,94 & 56,78 \\
\hline MAE & 103,03 & 27,89 & 78,86 & 63,60 & 70,88 & 47,94 & 66,52 & 36,96 & 41,05 & 49,11 \\
\hline \multirow[t]{3}{*}{ MAPE* } & 23,49 & 5,35 & 16,47 & 11,76 & 15,01 & 10,94 & 12,53 & 8,40 & 8,88 & 10,37 \\
\hline & \multicolumn{5}{|c|}{ Precipitação do outono } & \multicolumn{5}{|c|}{ Precipitação do inverno } \\
\hline & Norte & Sul & Noroeste & Sudoeste & Estado & Norte & Sul & Noroeste & Sudoeste & Estado \\
\hline r & 0,79 & 0,73 & 0,94 & 0,76 & 0,94 & 0,81 & 0,55 & 0,93 & 0,92 & 0,91 \\
\hline $\mathrm{R}_{\text {adj }}^{2}$ & 0,63 & 0,53 & 0,89 & 0,57 & 0,88 & 0,66 & 0,30 & 0,86 & 0,85 & 0,82 \\
\hline $\mathrm{Ea}$ & 14,87 & 17,73 & 12,54 & 14,19 & 20,25 & 13,57 & 38,74 & 13,19 & 15,55 & 32,09 \\
\hline Es & 71,66 & 53,50 & 73,76 & 52,46 & 64,33 & 75,81 & 46,33 & 79,28 & 100,13 & 74,13 \\
\hline EAmax & 102,93 & 109,83 & 96,49 & 86,27 & 158,86 & 134,86 & 128,04 & 116,09 & 145,66 & 145,66 \\
\hline MSE & 5356,8 & 3177,1 & 5597,5 & 2953,6 & 4548,7 & 5931,0 & 3647,8 & 6460,0 & 10267,5 & 6525,4 \\
\hline RMSE & 73,19 & 56,37 & 74,82 & 54,35 & 67,44 & 77,01 & 60,40 & 80,37 & 101,33 & 80,78 \\
\hline MAE & 71,33 & 52,31 & 73,69 & 51,63 & 64,27 & 71,68 & 50,06 & 77,38 & 98,77 & 74,20 \\
\hline MAPE* & 24,08 & 15,32 & 22,61 & 12,47 & 19,45 & 30,93 & 15,52 & 30,07 & 23,77 & 25,30 \\
\hline
\end{tabular}



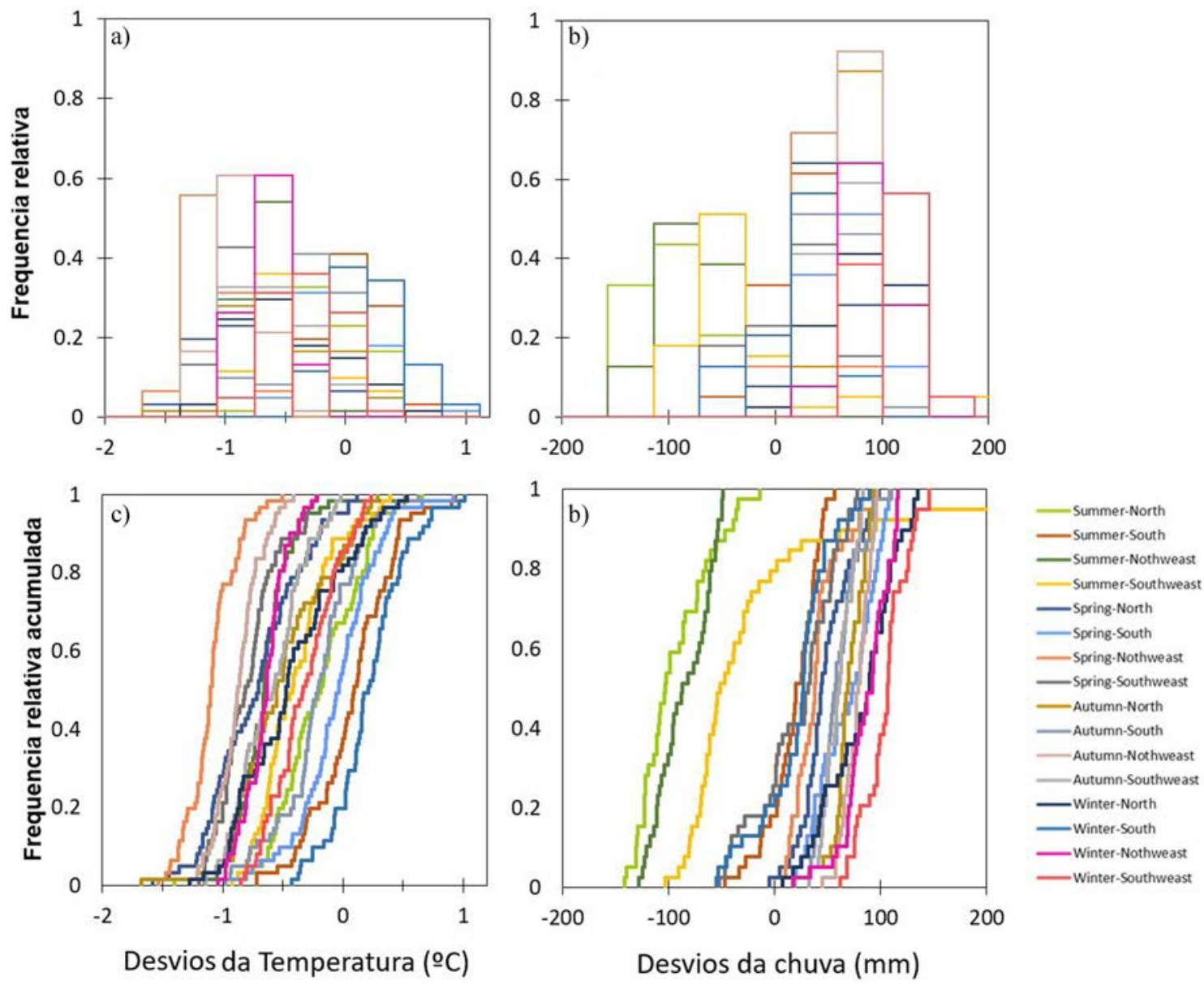

Figura 14 - Histogramas e frequência relativa acumulada dos desvios da temperatura e precipitação do ECMWF para o Estado do Paraná, Brasil. 

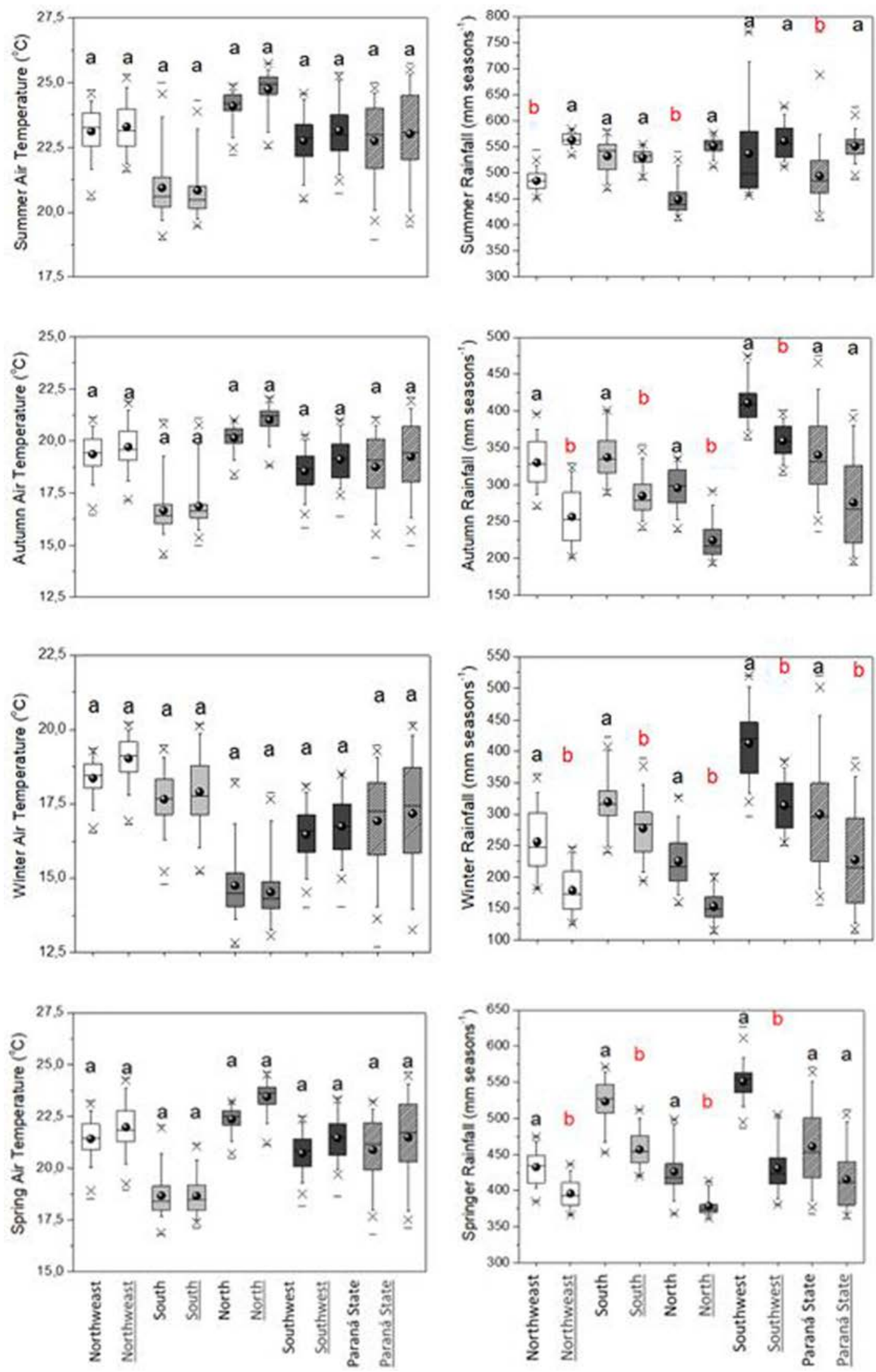

Figura 15 - Teste de Tukey ( $95 \%$ de confiabilidade) entre os dados oriundos da INMET (escrita preta) e ECMWF (escrita cinza sublinhada). 

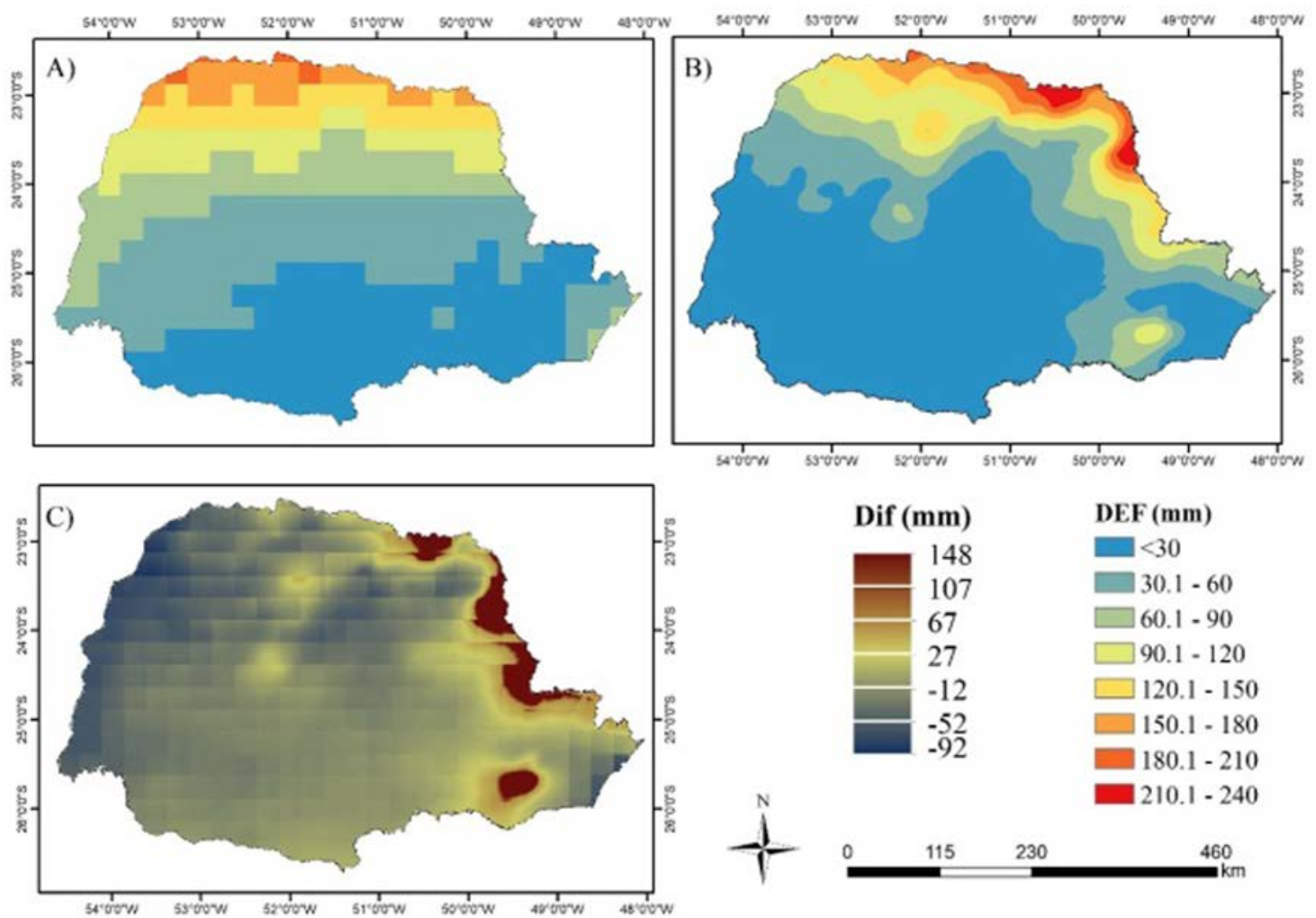

Figura 16 - Deficiência hídrica (mm) oriunda de dados do ECMWF (A) e dados do INMET (B) e os desvios entre as deficiências hídricas para o Estado do Paraná (C). Valores negativos na legenda indicam subestimativas feitas pelo ECMWF.

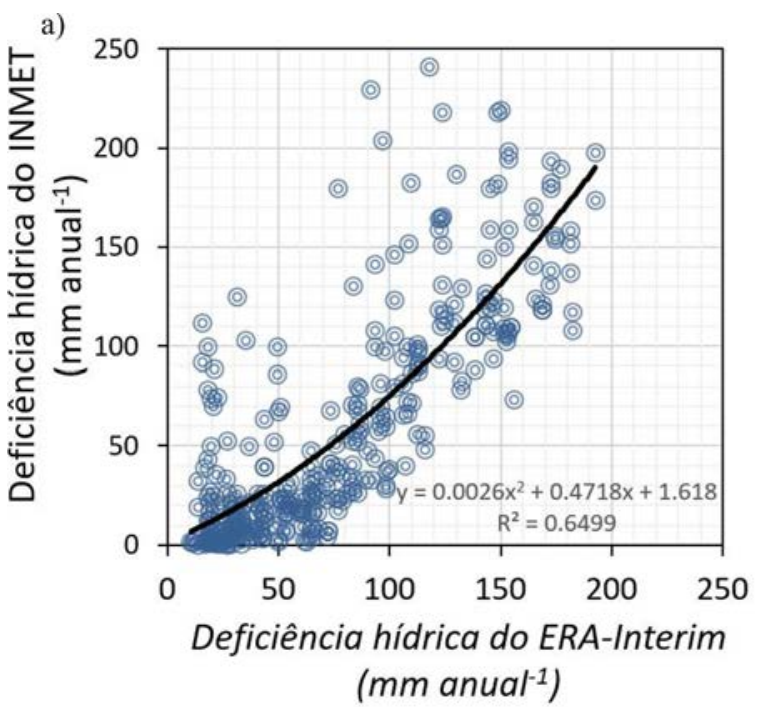

b)

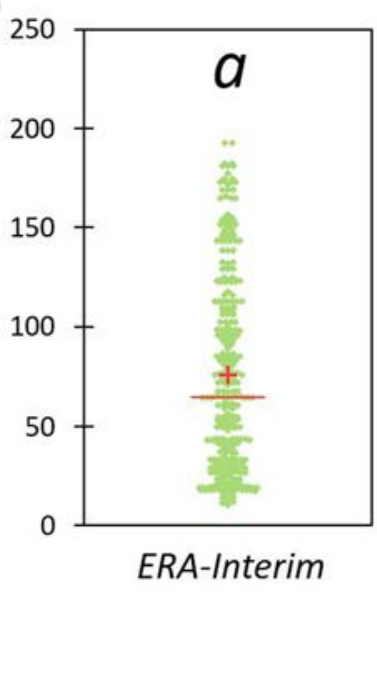

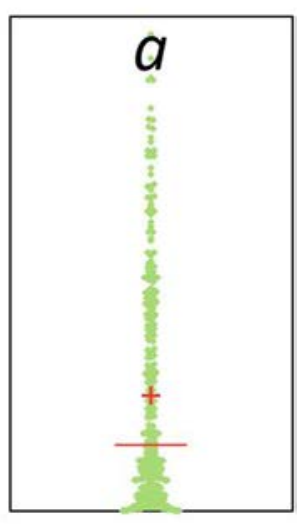

INMET

Figura 17 - Relação entre as deficiências hídricas oriundas dos dados meteorológicos de superfície e do ECMWF (A) e teste de Tukey a 95\% de confiabilidade comparando as deficiências hídricas do Estado do Paraná, Brasil.

\section{Conclusões}

A utilização de muitas estações meteorológicas virtuais como fonte dos dados previstos, possibilitou um mapeamento espacial acurado do conjunto de dados de reanálise do ERA-Interim em todas as estações do Estado do Paraná. A macrorregião com menor e maior erro para as estimativas do ERA-Interim são Sul e Noroeste para temperatura do ar e Sul e Norte para a precipitação. A temperatura do ar do ERA-Interim, na resolução padrão de $0,25^{\circ}$, demonstra ser acurado e viável na substituição dos 
dados de superfície para todas as regiões do estado de Paraná. Os dados da precipitação pluviométrica do ERAInterim demonstraram uma acurácia moderada, o que comprova a necessidade de uma correção para ser empregado, pois subestima a precipitação do verão e superestima as precipitações das demais estações do ano. A deficiência hídrica oriunda dos dados de reanálise não tem diferença significativa com a deficiência hídrica dos dados de superfície.

\section{Referências}

ALBUQUERQUE, A.H.P.; VIANA, T.V.A.; MARINHO, A.B.; SOUSA, G.G.; AZEVEDO, B.M. Irrigação e fertirrigação potássica na cultura da videira em condições semiáridas. Pesquisa Agropecuária Tropical, v. 43, n. 3, p. 315-321, 2013.

APARECIDO, L.E.D.O.; ROLIM, G.D.S.; RICHETTI, J.; SOUZA, P.S.D.; JOHANN, J.A. Köppen, Thornthwaite and Camargo climate classifications for climatic zoning in the State of Paraná, Brazil. Ciência e Agrotecnologia, v. 40, n. 4, p. 405-417, 2016.

AQUILA, V.; SWARTZ, W.H.; WAUGH, D.W.; COLARCO, P. R.; PAWSON, S.; POLVANI, L.M.; STOLARSKI, R.S. Isolating the roles of different forcing agents in global stratospheric temperature changes using model integrations with incrementally added single forcings. Journal of Geophysical Research: Atmospheres, v. 121, n. 13, p. 80678082, 2016.

BATTISTI, R.; SENTELHAS, P.C. Drought tolerance of brazilian soybean cultivars simulated by a simple agrometeorological yield model. Experimental Agriculture, v. 51, n. 2, p. 285-298, 2015.

BETTS, A. K.; BALL, J. H.; BELJAARS, A. C. M. Comparison between the land surface response of the ECMWF model and the FIFE-1987 data. Quarterly Journal of the Royal Meteorological Society, v. 119, n. 513, p. 975-1001, 1993.

BRIXNER, G.F.; SCHÖFFEL, E.R.; LAGO, I.; RADÜNZ, A.L.; KRÜGER, A.P. Risco de geada e duração dos subperíodos fenológicos da 'Cabernet Sauvignon' na região da Campanha. Revista Brasileira de Engenharia Agrícola e Ambiental, v. 18, n. 2, p. 217-224, 2014.

CANTELAUBE, PIERRE; TERRES, JEAN-MICHEL. Seasonal weather forecasts for crop yield modelling in Europe. Tellus A: Dynamic Meteorology and Oceanography, v. 57, n. 3, p. 476-487, 2005.

CEGLAR, A.; TORETI, A.; LECERF, R.; VAN DER VELDE, M.; DENTENER, F. Impact of meteorological drivers on regional inter-annual crop yield variability in France. Agricultural and forest meteorology, v. 216, p. 58-67, 2016.

COUTO, F.T.; SALGADO, R.; COSTA, M.J.; PRIOR, V. Precipitation in the Madeira Island over a 10-year period and the meridional water vapour transport during the winter seasons. International Journal of Climatology, v. 35, n. 13, p. 3748-3759, 2015.

CRAPARO, A.C.W.; VAN ASTEN, P.J.A.; LÄDERACH, P.; JASSOGNE, L.T.P.; GRABA S.W. Coffea arabica yields decline in Tanzania due to climate change: Global implica- tions. Agricultural and Forest Meteorology, v. 207, n. 1, p. 1-10. 2015.

DURAND, Y.; LATERNSER, M.; GIRAUD, G.; ETCHEVERS, P.; LESAFFRE, B.; MÉRINDOL, L. Reanalysis of $44 \mathrm{yr}$ of climate in the French Alps (1958-2002): methodology, model validation, climatology, and trends for air temperature and precipitation. Journal of Applied Meteorology and Climatology, v.48, n.3, p.429-449, 2009.

ECMWF. European Centre for Medium-Range Weather Forecasts, www.ecmwf.int. 2019.

FU, R.; BETTS, A.K. How well does the ERA40 surface water budget compare to observations in the Amazon River basin. Journal of Geophysical Research, v.13, n. D11, 2008.

GASPAR, N.A.; APARECIDO, L.E.O; ROLIM, G.S.; SOUZA, P.S.; BOTELHOS, T.G. Software for the management of weather stations and for agrometeorological calculations. Australian Journal of Crop Science, v. 9, n. 6, p. 545, 2015.

HOOGENBOOM, G. Contribution of agrometeorology to the simulation of crop production and its applications. Agricultural and Forest Meteorology, v. 103, n. 1-2, p. 137157, 2000.

KÖPPEN, W.; GEIGER, R. Klimate der Erde. Wall-map $150 \mathrm{~cm} \times 200 \mathrm{~cm}$. Gotha: Verlag Justus Perthes, 1928.

KRIGE, D.G. A statistical approach to some mine valuation and allied problems on the Witwatersrand: By DG Krige. 1951. Tese de Doutorado. University of the Witwatersrand.

MARENGO, J. A. Characteristics and spatio-temporal variability of the Amazon River Basin Water Budget. Climate Dynamics, v. 24, n. 1, p. 11-22, 2005.

MARTINS, E.; APARECIDO, L.E.O.; SANTOS, L.P.S.; MENDONÇA, J.M.A. de; SOUZA. P.S. Influência das condições climáticas na produtividade e qualidade do cafeeiro produzido na região do Sul de Minas Gerais. Coffee Science, v. 10, n. 4, p. 499-506, 2015.

MORAES, R.A.; ROCHA, J.V.; ROLIM, G.S.; LAMPARELLI, R.A.C.; MARTINS, M.M. Avaliação dos dados decendiais de precipitação e temperatura máxima e mínima do ar simulados pelo modelo ECMWF para o Estado de São Paulo. Irriga, v. 3, n. 17, p. 397-407, 2018.

MORAES, R.A.; ROCHA, J.V.; LAMPARELLI, R.A.C. Determination of total accumulated rainfall, global radiation, evapotranspiration and degree-days originated from the ECMWF model to sugar cane crop. Eng. Agríc., v. 34, n. 2, p. 322-331, 2014.

MORAES, R.A.; ROCHA, J.V.; ROLIM, G.; LAMPARELLI, R.A.C.; MARTINS, M. Evaluation of 10-day period precipitation, maximum and minimum air temperature data from the ECMWF model in sao paulo state. Brasilian Journal of Irrigation and Drainage, v. 17, n. 3 p. 397407, 2012.

NIU, X.; EASTERLING, W.; HAYS, C.J.; JACOBS, A.; MEARNS, L. Reliability and input-data induced uncertainty of the EPIC model to estimate climate change impact on sorghum yields in the U.S. Great Plains. Agriculture, Ecosystems and Environment, v. 129, n. 1-3, p. 268-276, 2008.

OLIVEIRA, R.A.; SANTOS, R.S.; RIBEIRO, A.; ZOLNIER, S.; BARBOSA, M.H. P. Estimativa da produtividade da cana- 
de-açúcar para as principais regiões produtoras de Minas Gerais usando-se o método ZAEI. Revista Brasileira Engenharia Agrícola e Ambiental, v. 16, n. 5, p. 549-557, 2012.

ORTH, R.; DUTRA, E.; TRIGO, I.F.; BALSAMO, G. Advancing land surface model development with satellite-based Earth observations. Hydrol. Earth Syst. Sci. Discuss., v. 21, n. 5, p. 2483-2495, 2017.

ORTH, R.; SENEVIRATNE, S.I. Using soil moisture forecasts for sub-seasonal summer temperature predictions in $\mathrm{Eu}-$ rope. Climate Dynamics, v. 43, n. 12, p. 3403-3418, 2014.

PEIXOTO, C.P.; CERQUEIRA, E.C.; SOARES FILHO, W.S.; CASTRO NETO, M.T.; LEDO, C.A.S. Análise de crescimento de diferentes genótipos de citros cultivados sob déficit hídrico. Revista Brasileira de Fruticultura, v. 28, n. 3, p. 439-443, 2006.

PEREIRA, A.R.; ANGELOCCI, L.R.; SENTELHAS, P.C. Agrometeorologia: fundamentos e aplicações práticas. Guaíba: Agropecuária, 478p, 2002.

PERSON, A.; GRAZZIANI, F. User guide to ECMWF forecast products. Meteorological Bulletin M3.2. ECMWF: Reading, 115 p., 2007.

RAHIMI, J.; EBRAHIMPOUR, M.; KHALILI, A. Spatial changes of extended De Martonne climatic zones affected by climate change in Iran. Theor Appl Climatol, v. 112, n. 34, p. 409-418, 2013.

RICCE, W.S.; CARVALHO, S.L.C.; CARAMORI, P.H.; AULER, P.A.M.; ROBERTO, S.R. Agroclimatic zoning for pineapple cultivation in the state of Paraná, Brazil. Semina: Ciências Agrárias, v. 35, n. 4, p. 2337-2346, 2014.

RODRIGUES, R.L.; MORETTO, A.C.; GUILHOTO, J.J.M. Productive and industry structure food in paraná, from 1980 to 1995. Revista de Economia e Agronegócio, v. 4, n. 1, p. 241-266, 2015.

SANTOS, R.F.; CARLESSO, R. Déficit hídrico e os processos morfológico e fisiológico das plantas. Revista Brasileira Engenharia Agrícola e Ambiental, v. 2, n. 3, p. 287-294, 1998.

SAPUCCI, L.F. Evaluation of Modeling Water-Vapor-Weighted Mean Tropospheric Temperature for GNSS-Integrated Water Vapor Estimates in Brazil. Journal of Applied Meteorology and Climatology, v. 53, n. 1, p. 715-730, 2014.

SODOUDI, S.; NOORIAN, A.; GEB, M.; REIMER, E. Daily precipitation forecast of ECMWF verified over Iran. Theoretical and Applied Climatology, v. 99, n. 1, p. 39-51, 2010.

WAGNER, M.V.; JADOSKI, S.O.; LIMA, A.S.; MAGGI, M.F.; POTT, C.A.; Avaliação do ciclo fenológico da cultura do milho em função da soma térmica em Guarapuava, Sul do Brasil. Revista Brasileira de Tecnologia Aplicada nas Ciências Agrárias, v. 4, n. 1, p. 135-149, 2011.

License information: This is an open-access article distributed under the terms of the Creative Commons Attribution License (type CC-BY), which permits unrestricted use, distribution and reproduction in any medium, provided the original article is properly cited. 